\title{
Early Onset of Efficacy Using a $1 \%$ and $2 \%$ Topical Minocycline Gel for the Treatment of Rosacea: a Small Open Label Study
}

\section{Bhatia N¹; Ahmadyar $\mathrm{M}^{1}$; Hansra $\mathrm{H}^{2}$; Del Rosso J3; Baldwin $\mathrm{H}^{4}$; Daniels $\mathrm{AM}^{2}$}

${ }^{1}$ Therapeutics Clinical Research, San Diego, CA; ${ }^{2}$ BioPharmX, Inc., Menlo Park, CA;

${ }^{3}$ JDR Dermatology Research, LLC, Las Vegas, NV; ${ }^{4}$ The Acne Treatment and Research Center, Morristown, NJ

\section{Introduction}

Rosacea is a common, chronic, and relapsing skin disorder that presents with a variety of clinical manifestations primarily on the central face ${ }^{1}$. The papulopustular subtype forms acne-like inflammatory papules, pustules, and plaques. Genetic, immune, inflammatory, vascular, and environmental mechanisms may contribute to its development 2,3 . Because no cure has been identified, current treatments are generally used chronically or intermittently and aim to suppress its symptoms ${ }^{4}$.

Minocycline is effective as a first-line systemic therapy for rosacea; it is thought that, like other tetracyclines, its antiinflammatory properties are responsible ${ }^{5}$. However, significant side effects such as gastrointestinal distress and vertigo may make long-term use of oral minocycline intolerable and chronic use may contribute to resistance. Another form of delivery with lower overall dosage and reduced systemic side effects is needed.

The study medication: Topical minocycline gel
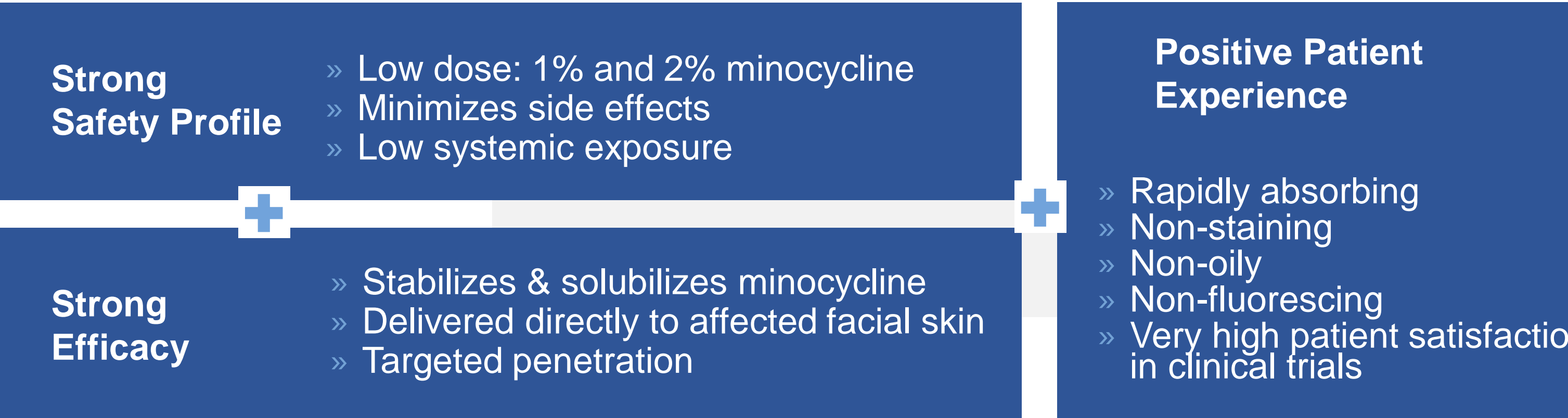

The study medication is the first completely solubilized minocycline gel for topical use. Its preliminary safety and efficacy profiles have been demonstrated in extensive preclinical testing. profiles have been demonstrated in extensive preclinical testing.
Additionally, it has completed phase $2 \mathrm{a}$ and $2 \mathrm{~b}$ testing for the treatment of acne vulgaris

\section{Methods}

This was a phase 2 open-label feasibility study of $1 \%$ and $2 \%$ formulations of a novel topical minocycline gel for the treatment of rosacea.

Open-label, single-site study

20 adults with moderate-to-severe (Grade 3 or 4 ; IGA*) papulopustular rosacea

»12 weeks of treatment evaluating 2 arms: $1 \%(n=10)$ and $2 \%$ ( $n=9$ [10 enrolled, 1 withdrawn]) formulations of topical minocycline gel

» Treatment assignment was non-randomized

»2-grade reduction in IGA to clear or almost clear ( 0 or 1 ) from baseline to 12 weeks

»Change in lesion count from baseline to 12 weeks

Cutaneous tolerability (4-point severity scales investigator- and subject-reported)

Hematology \& chemistry lab tests

" Treatment emergent AEs

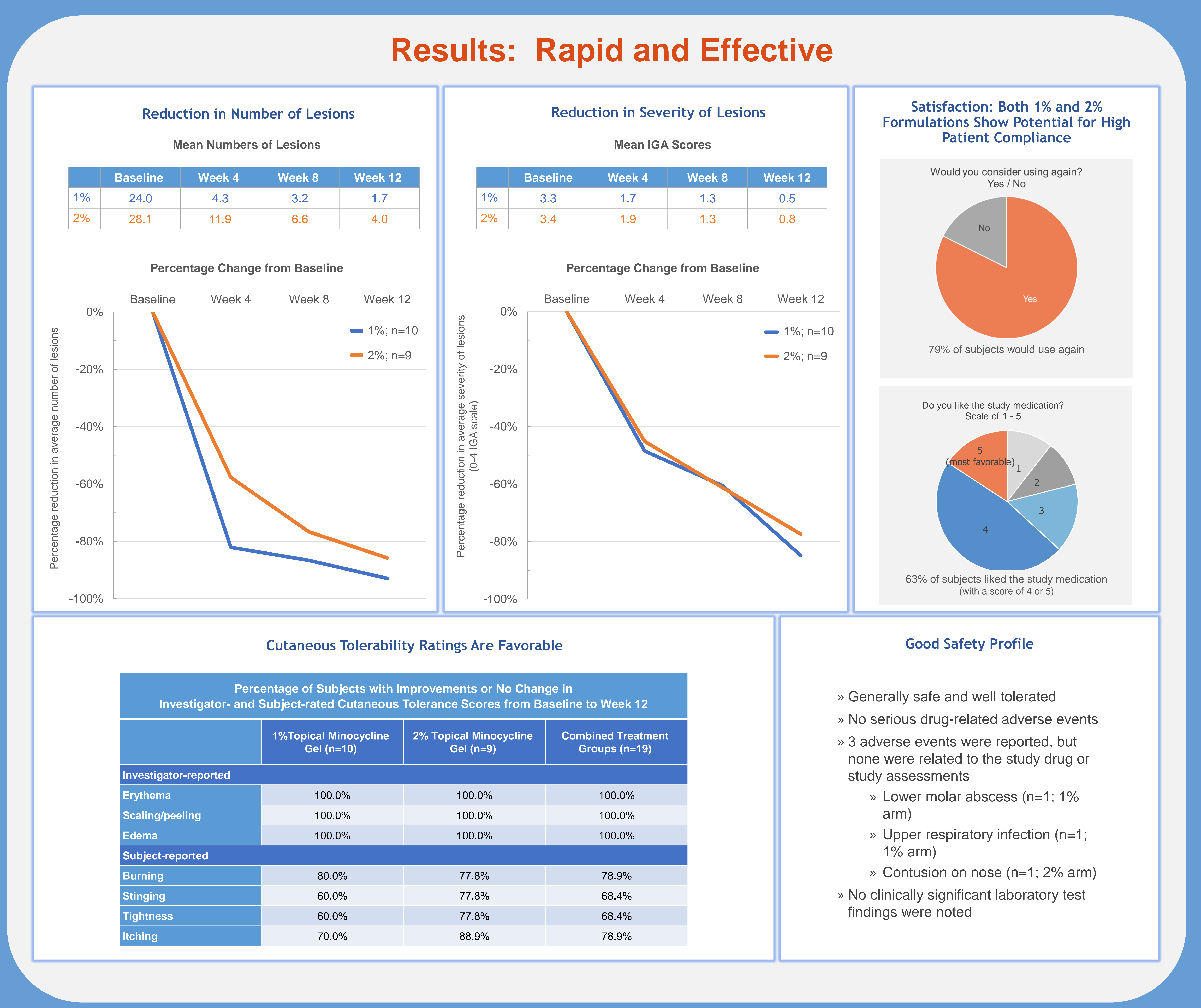

This is the first report of successful treatment of rosacea with a novel topical minocycline gel. The efficacy endpoints of reduction in number and severity of facial lesions were met and showed rapid onset. For both the $1 \%$ and $2 \%$ formulations, clinically meaningful improvements were reported after just four weeks of treatment.

Additionally, the minocycline gel treatment had a good profile for safety and tolerability. The majority of subjects stated they would use the minocycline gel again.

The study's small size and open-label single-center design must limit conclusions drawn but suggest that larger-scale testing is warranted. Next-phase clinical trials are planned.

1. Webster GFF Rosacea. Med Clin North Am 2009; 93:1183,
2. Abram K, Sim H, Maaroos HI. Oona M. Risk tactors ass

2. Abram K, Silm H, Maaroos HI, Oona M. Risk factors associated with rosacea. J Eur Acad Dermatol Venereol 2010; $24: 565$.
3. Wilkin J, Dahl M, Detmar M, Drake L, Feinstein A, Odom R, Powell F. Standard classification of rosacea: Report of the National Rosacea Society Expert Committee on the Classification and Staging of Rosacea. J Amer Acad Derm 2002; 46(4):584. 4. Maier LE. Management of rosacea. UpToDate January 2017.
5. Korting HC, Schollmann C. Tetracycline actions relevant to rosacea treatment. Skin Pharmacol Physiol 2009; 22:287.

\section{In conclusion,}

The rapid rate of improvement has the potential to improve treatment compliance and improved patient satisfaction.

An important advantage of the topica minocycline formulation - especially in chronic conditions such as rosacea in which long-term use is possible - may be in reduction of risks associated with systemic exposure to this antibiotic.

Sponsored and supported by BioPharmX Corporation (Menlo Park, CA).
The topical minocycline gel is limited by federal or US law to investigational use only. 